\title{
Effective Half-life
}

National Cancer Institute

\section{Source}

National Cancer Institute. Effective Half-life. NCI Thesaurus. Code C95007.

Time for the amount of a radionuclide in a living organism to be reduced to half of its value at a given initial time. The amount diminishes due to 2 concurrent processes: radioactive decay, and clearance from the system. 